\title{
$E P_{r}$ Solution to a System of Matrix Equations
}

\author{
Changzhou Dong, Yuping Zhang, Jianmin Song \\ School of Mathematics and Science, Shijiazhuang University of Economics, Shijiazhuang, China \\ Email: dongchangzh@sina.com, yuping.zh@163.com
}

Received September 29, 2013; revised October 29, 2013; accepted November 5, 2013

Copyright (C) 2013 Changzhou Dong et al. This is an open access article distributed under the Creative Commons Attribution License, which permits unrestricted use, distribution, and reproduction in any medium, provided the original work is properly cited.

\begin{abstract}
A square complex matrix $A$ is called $E P$ if it can be written in the form $U\left(A_{1} \oplus 0\right) U$ with $U$ being fixed unitary and $A_{1}$ being arbitrary matrix in $\mathbb{C}^{r \times r}$. We give necessary and sufficient conditions for the existence of the $E P_{r}$ solution to the system of complex matrix equation $A X=B, X C=D$ and present an expression of the $E P_{r}$ solution to the system when the solvability conditions are satisfied. In addition, the solution to an optimal approximation problem is obtained. Furthermore, the least square $E P_{r}$ solution with least norm to this system mentioned above is considered. The representation of such solution is also derived.
\end{abstract}

Keywords: EP Matrix; Matrix Equation; Moore-Penrose Inverse; Approximation Problem; Least Squares Solution

\section{Introduction}

Throughout we denote the complex $m \times n$ matrix space by $\mathbb{C}^{m \times n}$, the real $m \times n$ matrix space by $\mathbb{R}^{m \times n}$. The symbols $I, A^{*}, \mathcal{R}(A), \mathcal{N}(A)$, and $\|A\|$ stand for the identity matrix with the appropriate size, the conjugate transpose, the range, the null space, and the Frobenius norm of $A \in \mathbb{C}^{m \times n}$, respectively. The Moore-Penrose inverse of $A \in \mathbb{C}^{m \times n}$, denoted by $A^{\dagger}$ is defined to be the unique matrix $X \in \mathbb{C}^{n \times m}$ of the following matrix equations

$$
A X A=A, X A X=X,(X A)^{*}=X A,(A X)^{*}=A X .
$$

Recall that an $n \times n$ complex matrix $A$ is called $E P$ (or range Hermitian) if $A A^{\dagger}=A^{\dagger} A . \quad E P$ matrices were introduced by Schwerdtfeger in [1], ever since many authors have studied EP matrices with entries from complex number field to semigroups with involution and given various equivalent conditions and many characterizations for matrix to be EP (see, [2-5]).

Investigating the matrix equation

$$
A X=B
$$

with the unknown matrix $X$ being symmetric, reflexive, Hermitian-generalized Hamiltonian and re-positive definite is a very active research topic (see, [6-9]). As a generalization of (1), the classical system of matrix equations

$$
A X=C, X B=D
$$

has attracted many people's attention and many results have been obtained about system (2) with various constraints, such as bisymmetric, Hermitian, positive semidefinite, reflexive, and generalized reflexive solutions, and so on (see, [9-12]). It is well-known that EP matrices are a wide class of objects that include many matrices as their special cases, such as Hermitian and skewHermitian matrices (i.e., $A^{*}= \pm A$ ), normal matrices (i.e., $A A^{*}=A^{*} A$ ), as well as all nonsingular matrices. Therefore investigating the EP solution of the matrix Equation (2) is very meaningful.

Pearl showed in ([2]) that a matrix $A$ is $E P$ if and only if it can be written in the form $U\left(A_{1} \oplus 0\right) U^{*}$ with $U$ unitary and $A_{1}$ nonsingular. A square complex matrix $A$ is called $E P_{r}$ if it can be written in the form $U\left(A_{1} \oplus 0\right) U^{*}$, where $U$ is fixed unitary and $A_{1}$ is arbitrary matrix in $\mathbb{C}^{r \times r}$. To our knowledge, so far there has been little investigation of this $E P_{r}$ solution to (2).

Motivated by the work mentioned above, we investigate $E P_{r}$ solution to (2). We also consider the optimal approximation problem

$$
\|\hat{X}-E\|=\min _{X \in S_{X}}\|X-E\|,
$$

where $E$ is a given matrix in $\mathbb{C}^{n \times n}$ and $S_{X}$ the set of all $E P_{r}$ solutions to (2). In many case Equation (2) has not an $E P_{r}$ solution. Hence we need to further study its 
least squares solution, which can be described as follows: Let $\mathbb{C}_{e p}^{n \times n}(U)$ denote the set of all $E P_{r}$ matrices with fixed unitary matrix $U$ in $\mathbb{C}^{n \times n}$,

$$
S_{L}=\left\{X \mid \min _{X \in \mathbb{C}_{e p}^{n \times n}(U)}\|A X-C\|^{2}+\|X B-D\|^{2}\right\} .
$$

Find $\tilde{X} \in \mathbb{C}_{e p}^{n \times n}(U)$ such that

$$
\|\tilde{X}\|=\min _{X \in S_{L}}\|X\| \text {. }
$$

In Section 2, we present necessary and sufficient conditions for the existence of the EP $P_{r}$ solution to (2), and give an expression of this solution when the solvability conditions are met. In Section 3, we derive an optimal approximation solution to (3). In Section 4, we provide the least squares $E P_{r}$ solution to (4).

\section{2. $E P_{r}$ Solution to (2)}

In this section, we establish the solvability conditions and the general expression for the $E P_{r}$ solution to (2).

Throughout we denotes $\mathbb{C}_{e p}^{n \times n}(U)$ the set of all $E P_{r}$ matrices with fixed unitary matrix $U$ in $\mathbb{C}^{n \times n}$, i.e.,

$$
\mathbb{C}_{e p}^{n \times n}=\left\{A \mid A=U\left(A_{1} \oplus 0\right) U^{*}\right\},
$$

where $U$ is fixed unitary and $A_{1}$ is arbitrary matrix in $\mathbb{C}^{r \times r}$.

Lemma 2.1. ([3]) Let $A, C \in \mathbb{C}^{m \times n}, B, D \in \mathbb{C}^{n \times p}$. Then the system of matrix equations $A X=C, X B=D$ is consistent if and only if

$$
\mathcal{R}(C) \subset \mathcal{R}(A), \mathcal{N}(B) \subset \mathcal{N}(D), A D=C B .
$$

In that case, the general solution of this system is

$$
X=A^{\dagger} C+D B^{\dagger}-A^{\dagger} A D B^{\dagger}+\left(I-A^{\dagger} A\right) Y\left(I-B B^{\dagger}\right) \text {, }
$$

where $Y \in \mathbb{C}^{n \times n}$ is arbitrary.

Now we consider the $E P_{r}$ solution to (1). By the definition of $E P_{r}$ matrix, the solution has the following factorization:

$$
X=U\left[\begin{array}{cc}
X_{1} & 0 \\
0 & 0
\end{array}\right] U^{*}
$$

Let

$$
\begin{gathered}
A U=\left[A_{1}, A_{2}\right], \quad C U=\left[C_{1}, C_{2}\right], \\
U^{*} B=\left[\begin{array}{l}
B_{1} \\
B_{2}
\end{array}\right], \quad U^{*} D=\left[\begin{array}{l}
D_{1} \\
D_{2}
\end{array}\right],
\end{gathered}
$$

where $A_{1}, C_{1} \in \mathbb{C}^{m \times r}, \quad A_{2}, C_{2} \in \mathbb{C}^{m \times(n-r)}, \quad B_{1}, D_{1} \in \mathbb{C}^{r \times p}$, $B_{2}, D_{2} \in \mathbb{C}^{(n-r) \times p}$, then (2) has $E P_{r}$ solution if and only if the system of matrix equations

$$
A_{1} X_{1}=C_{1}, X_{1} B_{1}=D_{1}, 0=C_{2}, 0=D_{2},
$$

is consistent. By Lemma 2.1, we have the following theorem.

Theorem 2.2. Let $A, C \in \mathbb{C}^{m \times n}, B, D \in \mathbb{C}^{n \times p}$ and

$$
A U=\left[A_{1}, A_{2}\right], \quad U^{*} B=\left[\begin{array}{l}
B_{1} \\
B_{2}
\end{array}\right], \quad U^{*} D=\left[\begin{array}{c}
D_{1} \\
D_{2}
\end{array}\right],
$$

where $A_{1}, C_{1} \in \mathbb{C}^{m \times r}, A_{2}, C_{2} \in \mathbb{C}^{m \times(n-r)}$,

$$
B_{1}, D_{1} \in \mathbb{C}^{r \times p}, B_{2}, D_{2} \in \mathbb{C}^{(n-r) \times p} \text {. }
$$

Then the matrix Equation (2) has a $E P_{r}$ solution in $\mathbb{C}^{n \times n}$ if and only if

$$
\begin{aligned}
& \mathcal{R}\left(C_{1}\right) \subset \mathcal{R}\left(A_{1}\right), \mathcal{N}\left(B_{1}\right) \subset \mathcal{N}\left(D_{1}\right), \\
& A_{1} D_{1}=C_{1} B_{1}, C_{2}=D_{2}=0 .
\end{aligned}
$$

In that case, the general $E P_{r}$ solution of (1) is

$$
X=U\left[\begin{array}{cc}
A_{1}^{\dagger} C_{1}+D_{1} B_{1}^{\dagger}-A_{1}^{\dagger} A_{1} D_{1} B_{1}^{\dagger}+\left(I-A_{1}^{\dagger} A_{1}\right) Y_{1}\left(I-B_{1} B_{1}^{\dagger}\right) & 0 \\
0 & 0
\end{array}\right] U^{*},
$$

where $Y_{1} \in \mathbb{C}^{r \times r}$ is arbitrary.

\section{The Solution of Optimal Approximation Problem (3)}

When the set $S_{X}$ of all $E P_{r}$ solution to (2) is nonempty, it is easy to verify $S_{X}$ is a closed set. Therefore the optimal approximation problem (3) has a unique solution by [13]. We first verify the following lemma.

Lemma 3.1. Let $A \in \mathbb{C}^{m \times n}, B \in \mathbb{C}^{n \times p}, C \in \mathbb{C}^{n \times n}$. Then the procrustes problem

$$
\min _{X \in \mathbb{C}^{n \times n}}\left\|\left(I-A^{\dagger} A\right) X\left(I-B B^{\dagger}\right)-C\right\|
$$

has a solution which can be expressed as

$$
X=C+A^{\dagger} G_{1}+G_{2} B^{\dagger},
$$

where $G_{1} \in \mathbb{C}^{m \times n}, G_{2} \in \mathbb{C}^{n \times p}$ are arbitrary matrices.

Proof. It follows from the properties of Moore-Penrose generalized inverse and the inner product that

$$
\begin{aligned}
& \left\|\left(I-A^{\dagger} A\right) X\left(I-B B^{\dagger}\right)-C\right\|^{2}=\left\langle\left(I-A^{\dagger} A\right) X\left(I-B B^{\dagger}\right)-C,\left(I-A^{\dagger} A\right) X\left(I-B B^{\dagger}\right)-C\right\rangle \\
& =\left\langle\left(I-A^{\dagger} A\right)(X-C)\left(I-B B^{\dagger}\right),\left(I-A^{\dagger} A\right)(X-C)\left(I-B B^{\dagger}\right)\right\rangle+\left\langle\left(I-A^{\dagger} A\right) C\left(I-B B^{\dagger}\right),\left(I-A^{\dagger} A\right) C\left(I-B B^{\dagger}\right)\right\rangle \\
& =\left\|\left(I-A^{\dagger} A\right)(X-C)\left(I-B B^{\dagger}\right)\right\|^{2}+\left\|\left(I-A^{\dagger} A\right) C\left(I-B B^{\dagger}\right)\right\|^{2} .
\end{aligned}
$$


Hence,

$$
\min _{X \in \mathbb{C}^{n \times n}}\left\|\left(I-A^{\dagger} A\right) X\left(I-B B^{\dagger}\right)-C\right\|
$$

if and only if

$$
\min _{X \in \mathbb{C}^{n \times n}}\left\|\left(I-A^{\dagger} A\right)(X-C)\left(I-B B^{\dagger}\right)\right\| .
$$

It is clear that $X=C+A^{\dagger} G_{1}+G_{2} B^{\dagger}$ with $G_{1} \in \mathbb{C}^{m \times n}, \quad G_{2} \in \mathbb{C}^{n \times p}$ are arbitrary is the solution of the above procrustes problem.

Theorem 3.2. Let $A, C \in \mathbb{C}^{m \times n}, B, D \in \mathbb{C}^{n \times p}, E \in \mathbb{C}^{n \times n}$, and

$$
U^{*} E U=\left[\begin{array}{ll}
E_{1} & E_{2} \\
E_{3} & E_{4}
\end{array}\right],
$$

where $E_{1} \in \mathbb{C}^{r \times r}$. Assume $S_{X}$ is nonempty, then the optimal approximation problem (3) has a unique solution $\hat{X}$ and

$$
\hat{X}=U\left[\begin{array}{cc}
A_{1}^{\dagger} C_{1}+D_{1} B_{1}^{\dagger}-A_{1}^{\dagger} A_{1} D_{1} B_{1}^{\dagger}+\left(I-A_{1}^{\dagger} A_{1}\right) E_{1}\left(I-B_{1} B_{1}^{\dagger}\right) & 0 \\
0 & 0
\end{array}\right] U^{*},
$$

Proof. Since $S_{X}$ is nonempty, $X \in S_{X}$ has the form of (6). It follows from (7) and the unitary invariance of

Frobenius norm that

$$
\begin{aligned}
& \|X-E\|^{2} \\
= & \left\|U\left[\begin{array}{cc}
A_{1}^{\dagger} C_{1}+D_{1} B_{1}^{\dagger}-A_{1}^{\dagger} A_{1} D_{1} B_{1}^{\dagger}+\left(I-A_{1}^{\dagger} A_{1}\right) Y_{1}\left(I-B_{1} B_{1}^{\dagger}\right) & 0 \\
0 & 0
\end{array}\right] U^{*}-C\right\|^{2} \\
= & \left\|\left[\begin{array}{cc}
A_{1}^{\dagger} C_{1}+D_{1} B_{1}^{\dagger}-A_{1}^{\dagger} A_{1} D_{1} B_{1}^{\dagger}+\left(I-A_{1}^{\dagger} A_{1}\right) Y_{1}\left(I-B_{1} B_{1}^{\dagger}\right) & 0 \\
0 & 0
\end{array}\right]-U^{*} C U\right\|^{2} \\
= & \left\|\left[\begin{array}{cc}
A_{1}^{\dagger} C_{1}+D_{1} B_{1}^{\dagger}-A_{1}^{\dagger} A_{1} D_{1} B_{1}^{\dagger}+\left(I-A_{1}^{\dagger} A_{1}\right) Y_{1}\left(I-B_{1} B_{1}^{\dagger}\right) & 0 \\
0 & 0
\end{array}\right]-\left[\begin{array}{cc}
E_{1} & E_{2} \\
E_{3} & E_{4}
\end{array}\right]\right\|^{2} \\
= & \left\|\left(I-A_{1}^{\dagger} A_{1}\right) Y_{1}\left(I-B_{1} B_{1}^{\dagger}\right)-\left(E_{1}+A_{1}^{\dagger} A_{1} D_{1} B_{1}^{\dagger}-A_{1}^{\dagger} C_{1}-D_{1} B_{1}^{\dagger}\right)\right\|^{2} \\
& +\left\|E_{2}\right\|^{2}+\left\|E_{3}\right\|^{2}+\left\|E_{4}\right\|^{2} .
\end{aligned}
$$

Therefore, there exists $\hat{X} \in S_{X}$ such that the matrix such that nearness problem (3) holds if and only if exist $Y_{1} \in \mathbb{C}^{r \times r}$

$$
\min _{Y_{1}}\left\|\left(I-A_{1}^{\dagger} A_{1}\right) Y_{1}\left(I-B_{1} B_{1}^{\dagger}\right)-\left(E_{1}+A_{1}^{\dagger} A_{1} D_{1} B_{1}^{\dagger}-A_{1}^{\dagger} C_{1}-D_{1} B_{1}^{\dagger}\right)\right\| .
$$

According to Lemma 3.1, we have

$$
Y_{1}=E_{1}+A_{1}^{\dagger} A_{1} D_{1} B_{1}^{\dagger}-A_{1}^{\dagger} C_{1}-D_{1} B_{1}^{\dagger}+A_{1}^{\dagger} G_{1}+G_{2} B_{1}^{\dagger},
$$

where $G_{1} \in \mathbb{C}^{m \times r}, G_{2} \in \mathbb{C}^{r \times p}$ are arbitrary. Substituting $Y_{1}$ into (6), we obtain that the solution of the matrix nearness problem (3) can be expressed as (8).

\section{The Least Squares $E P_{r}$ Solution to (4)}

In this section, we give the explicit expression of the least squares $E P_{r}$ solution to (4).

Lemma 4.1. ([12]) Given $E, F \in \mathbb{C}^{m \times n}$, $\Omega_{1}=\operatorname{diag}\left(a_{1}, \cdots, a_{m}\right), \quad \Omega_{2}=\operatorname{diag}\left(b_{1}, \cdots, b_{n}\right)$, $a_{i}>0(i=1, \cdots, m), \quad b_{j}>0(j=1, \cdots, n)$. Then there exists a unique matrix $\vec{S} \in \mathbb{C}^{m \times n}$ such that

$$
\left\|\Omega_{1} S-E\right\|^{2}+\left\|S \Omega_{2}-F\right\|^{2}=\min .
$$

And $\bar{S}$ can be expressed as

$$
\bar{S}=\varnothing *\left(\Omega_{1} E+F \Omega_{2}\right),
$$

where $\varnothing=\left(\frac{1}{a_{i}^{2}+b_{j}^{2}}\right) \in \mathbb{R}^{m \times n}$.

Theorem 4.2. Let $A, C \in \mathbb{C}^{m \times n}, B, D \in \mathbb{C}^{n \times p}$ and

$$
\begin{gathered}
A U=\left[A_{1}, A_{2}\right], C U=\left[C_{1}, C_{2}\right], \\
U^{*} B=\left[\begin{array}{l}
B_{1} \\
B_{2}
\end{array}\right], U^{*} D=\left[\begin{array}{l}
D_{1} \\
D_{2}
\end{array}\right],
\end{gathered}
$$

where $A_{1}, C_{1} \in \mathbb{C}^{m \times r}, \quad A_{2}, C_{2} \in \mathbb{C}^{m \times(n-r)}, \quad B_{1}, D_{1} \in \mathbb{C}^{r \times p}$, 
$B_{2}, D_{2} \in \mathbb{C}^{(n-r) \times p}$. Assume that the singular value decomposition of $A_{1}, B_{1}$ are as follows

$$
A_{1}=W\left[\begin{array}{cc}
M_{1} & 0 \\
0 & 0
\end{array}\right] V^{*}, B_{1}=P\left[\begin{array}{cc}
N_{1} & 0 \\
0 & 0
\end{array}\right] Q^{*},
$$

where $W=\left[W_{1}, W_{2}\right] \in \mathbb{C}^{m \times m}, \quad V=\left[V_{1}, V_{2}\right] \in \mathbb{C}^{r \times r}$,
$P=\left[P_{1}, P_{2}\right] \in \mathbb{C}^{r \times r}$ and $Q=\left[Q_{1}, Q_{2}\right] \in \mathbb{C}^{p \times p}$ are unitary matrices, $M_{1}=\operatorname{diag}\left(\sigma_{1}, \cdots, \sigma_{r_{1}}\right), \quad \sigma_{i}>0\left(i=1, \cdots, r_{1}\right)$, $r_{1}=\operatorname{rank}\left(M_{1}\right), W_{1} \in \mathbb{C}^{m \times r_{1}}, \quad V_{1} \in \mathbb{C}^{r \times r_{1}}$,

$N_{1}=\operatorname{diag}\left(\rho_{1}, \cdots, \rho_{r_{2}}\right), \quad \rho_{j}>0\left(j=1, \cdots, r_{2}\right)$,

$r_{2}=\operatorname{rank}\left(N_{1}\right), \quad P_{1} \in \mathbb{C}^{r \times r_{2}}, \quad Q_{1} \in \mathbb{C}^{p \times r_{2}}$. Then $X \in S_{L}$ can be expressed as

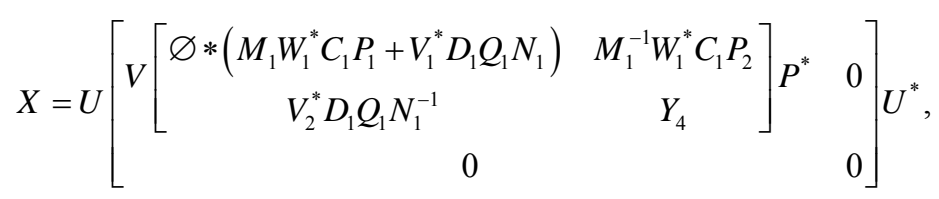

where $\varnothing=\left(\frac{1}{\sigma_{i}^{2}+\rho_{j}^{2}}\right) \in \mathbb{R}^{r_{1} \times r_{2}}$, and $Y_{4} \in \mathbb{C}^{\left(r-r_{1}\right) \times\left(r-r_{2}\right)}$ is

an arbitrary matrix.

Proof. It yields from (9) that

$$
\begin{aligned}
\|A X-C\|^{2}+\|X B-D\|^{2} & =\left\|A U\left[\begin{array}{cc}
X_{1} & 0 \\
0 & 0
\end{array}\right] U^{*}-C\right\|^{2}+\left\|U\left[\begin{array}{cc}
X_{1} & 0 \\
0 & 0
\end{array}\right] U^{*} B-D\right\|^{2} \\
& =\left\|\left[A_{1}, A_{2}\right]\left[\begin{array}{cc}
X_{1} & 0 \\
0 & 0
\end{array}\right]-\left[C_{1}, C_{2}\right]\right\|\left\|^{2}+\right\|\left[\begin{array}{cc}
X_{1} & 0 \\
0 & 0
\end{array}\right]\left[\begin{array}{c}
B_{1} \\
B_{2}
\end{array}\right]-\left[\begin{array}{c}
D_{1} \\
D_{2}
\end{array}\right] \|^{2} \\
& =\left\|A_{1} X_{1}-C_{1}\right\|^{2}+\left\|X_{1} B_{1}-D_{1}\right\|^{2}+\left\|C_{2}\right\|^{2}+\left\|D_{2}\right\|^{2} \\
& =\left\|W\left[\begin{array}{cc}
M_{1} & 0 \\
0 & 0
\end{array}\right] V^{*} X_{1}-C_{1}\right\|^{2}+\left\|X_{1} P\left[\begin{array}{cc}
N_{1} & 0 \\
0 & 0
\end{array}\right] Q^{*}-D_{1}\right\|^{2}+\left\|C_{2}\right\|^{2}+\left\|D_{2}\right\|^{2} \\
& =\left\|\left[\begin{array}{cc}
M_{1} & 0 \\
0 & 0
\end{array}\right] V^{*} X_{1} P-W^{*} C_{1} P\right\|^{2}+\left\|V^{*} X_{1} P\left[\begin{array}{cc}
N_{1} & 0 \\
0 & 0
\end{array}\right]-V^{*} D_{1} Q\right\|^{2}+\left\|C_{2}\right\|^{2}+\left\|D_{2}\right\|^{2} .
\end{aligned}
$$

Assume that

$$
V^{*} X_{1} P=\left(\begin{array}{ll}
Y_{1} & Y_{2} \\
Y_{3} & Y_{4}
\end{array}\right), Y_{1} \in \mathbb{C}^{r_{1} \times r_{2}}, Y_{4} \in \mathbb{C}^{\left(r-r_{1}\right) \times\left(r-r_{2}\right)} .
$$

Then we have

$$
\begin{aligned}
\| & A X-C\left\|^{2}+\right\| X B-D \|^{2} \\
= & \left\|M_{1} Y_{1}-W_{1}^{*} C_{1} P_{1}\right\|^{2}+\left\|Y_{1} N_{1}-V_{1}^{*} D_{1} Q_{1}\right\|^{2} \\
& +\left\|M_{1} Y_{2}-W_{1}^{*} C_{1} P_{2}\right\|^{2}+\left\|Y_{3} N_{1}-V_{2}^{*} D_{1} Q_{1}\right\|^{2}+\left\|W_{2}^{*} C_{1} P_{1}\right\|^{2} \\
& +\left\|W_{2}^{*} C_{1} P_{2}\right\|^{2}+\left\|V_{1}^{*} D_{1} Q_{2}\right\|^{2}+\left\|V_{2}^{*} D_{1} Q_{2}\right\|^{2}+\left\|C_{2}\right\|^{2}+\left\|D_{2}\right\|^{2} .
\end{aligned}
$$

Hence

$$
\min _{X \in \mathbb{C}_{e p}^{n \times n}(U)}\|A X-C\|^{2}+\|X B-D\|^{2}
$$

is solvable if and only if there exist $Y_{1}, Y_{2}, Y$ such that

$$
\begin{gathered}
\left\|M_{1} Y_{1}-W_{1}^{*} C_{1} P_{1}\right\|^{2}+\left\|Y_{1} N_{1}-V_{1}^{*} D_{1} Q_{1}\right\|^{2}=\min , \\
\left\|M_{1} Y_{2}-W_{1}^{*} C_{1} P_{2}\right\|^{2}=\min ,\left\|Y_{3} N_{1}-V_{2}^{*} D_{1} Q_{1}\right\|^{2}=\min .
\end{gathered}
$$

It follows from (12) and (13) that

$$
\begin{gathered}
Y_{1}=\varnothing *\left(M_{1} W_{1}^{*} C_{1} P_{1}+V_{1}^{*} D_{1} Q_{1} N_{1}\right), \\
Y_{2}=M_{1}^{-1} W_{1}^{*} C_{1} P_{2}, Y_{3}=V_{2}^{*} D_{1} Q_{1} N_{1}^{-1}
\end{gathered}
$$

where $\varnothing=\left(\frac{1}{\sigma_{i}^{2}+\rho_{j}^{2}}\right) \in \mathbb{R}^{r_{1} \times r_{2}}$. Substituting (14) and (15) into (11), we can get the form of elements in $S_{L}$ is (10).

Theorem 4.3. Assume the notations and conditions are the same as Theorem 4.2. Then

$$
\|\tilde{X}\|=\min _{X \in S_{L}}\|X\|
$$

if and only if

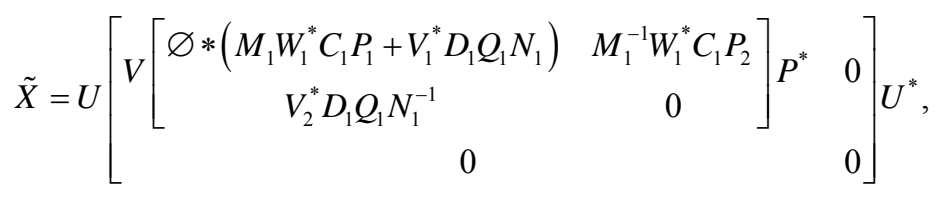


where $\varnothing=\left(\frac{1}{\sigma_{i}^{2}+\rho_{j}^{2}}\right) \in \mathbb{R}^{r_{1} \times r_{2}}$.

Proof. In Theorem 4.2, it implies from (10) that $\min _{X \in S_{L}}\|X\|$ is equivalent to $X$ has the expression (10) with $Y_{4}=0$. Hence (16) holds.

\section{Acknowledgements}

This research was supported by the Natural Science Foundation of Hebei province (A2012403013), the Natural Science Foundation of Hebei province (A2012205028) and the Education Department Foundation of Hebei province (Z2013110).

\section{REFERENCES}

[1] H. Schwerdtfeger, "Introduction to Linear Algebra and the Theory of Matrices," P. Noordhoff, Groningen, 1950.

[2] M. H. Pearl, "On normal and EP matrices," Michigan Mathematical Journal, Vol. 6, No. 1, 1959, pp. 1-5. http://dx.doi.org/10.1307/mmj/1028998132

[3] C. R. Rao and S. K. Mitra, "Generalized Inverse of Matrices and Its Applications," Wiley, New York, 1971.

[4] O. M. Baksalary and G. Trenkler, "Characterizations of EP, normal, and Hermitian matrices," Linear Multilinear Algebra, Vol. 56, 2008, pp. 299-304.

http://dx.doi.org/10.1080/03081080600872616

[5] Y. Tian and H. X. Wang, "Characterizations of EP Matrices and Weighted-EP Matrices," Linear Algebra Applications, Vol. 434, No. 5, 2011, pp. 1295-1318. http://dx.doi.org/10.1016/j.laa.2010.11.014

[6] K.-W. E. Chu, "Singular Symmetric Solutions of Linear Matrix Equations by Matrix Decompositions," Linear Al- gebra Applications, Vol. 119, 1989, pp. 35-50.

http://dx.doi.org/10.1016/0024-3795(89)90067-0

[7] R. D. Hill, R. G. Bates and S. R. Waters, "On Centrohermitian Matrices," SIAM Journal on Matrix Analysis and Applications, Vol. 11, No. 1, 1990, pp. 128-133. http://dx.doi.org/10.1137/0611009

[8] Z. Z. Zhang, X. Y. Hu and L. Zhang, "On the HermitianGeneralized Hamiltonian Solutions of Linear Mattrix Equations," SIAM Journal on Matrix Analysis and Applications, Vol. 27, No. 1, 2005, pp. 294-303. http://dx.doi.org/10.1137/S0895479801396725

[9] A. Dajić and J. J. Koliha, "Equations $a x=c$ and $x b=d$ in Rings and Rings with Involution with Applications to Hilbert Space Operators," Linear Algebra Applications, Vol. 429, No. 7, 2008, pp. 1779-1809. http://dx.doi.org/10.1016/j.laa.2008.05.012

[10] C. G. Khatri and S. K. Mitra, "Hermitian and Nonnegative Definite Solutions of Linear Matrix Equations," SIAM Journal on Matrix Analysis and Applications, Vol. 31, No. 4, 1976, pp. 579-585. http://dx.doi.org/10.1137/0131050

[11] F. J. H. Don, "On the Symmetric Solutions of a Linear Matrix Equation," Linear Algebra Applications, Vol. 93, 1987, pp. 1-7.

http://dx.doi.org/10.1016/S0024-3795(87)90308-9

[12] H. X. Chang, Q. W. Wang and G. J. Song, "(R,S)-Conjugate Solution to a Pair of Linear Matrix Equations," Applied Mathematics and Computation, Vol. 217, 2010, pp. 73-82. http://dx.doi.org/10.1016/j.amc.2010.04.053

[13] E. W. Cheney, "Introduction to Approximation Theory," McGraw-Hill Book Co., 1966. 